Nonlinear Processes in Geophysics, 12, 515-525, 2005

SRef-ID: $1607-7946 / \mathrm{npg} / 2005-12-515$

European Geosciences Union

(c) 2005 Author(s). This work is licensed

under a Creative Commons License.

\title{
Nonlinear data-assimilation using implicit models
}

\author{
A. D. Terwisscha van Scheltinga ${ }^{1}$ and H. A. Dijkstra ${ }^{2,1}$ \\ ${ }^{1}$ Institute for Marine and Atmospheric research Utrecht, Department of Physics and Astronomy, Utrecht University, The \\ Netherlands \\ ${ }^{2}$ Department of Atmospheric Science, Colorado State University, Fort Collins, CO, USA
}

Received: 16 September 2004 - Revised: 21 February 2005 - Accepted: 5 April 2005 - Published: 19 May 2005

Part of Special Issue "Nonlinear processes in solar-terrestrial physics and dynamics of Earth-Ocean-System"

\begin{abstract}
We show how the traditional 4D-Var method can be adapted for implicit time-integration and extended for multi-parameter estimation. We present the algorithm for this new method, which we call I4D-Var, and demonstrate its performance using a fully-implicit barotropic quasigeostrophic model of the wind-driven double-gyre ocean circulation. For the latter model, the different regimes of flow behavior and the regime boundaries (i.e. bifurcation points) are well known and hence the parameter estimation problem can be systematically studied. It turns out that I4D-Var is able to correctly estimate parameter values, even when background flow and "observations" are in different dynamical regimes.
\end{abstract}

\section{Introduction}

The kinetic energy of ocean flows is distributed over many scales of motion. In a numerical model with a specified resolution, only part of the range of scales can be resolved. The effect of the unresolved scales on the transport of momentum, heat and salt are represented by so-called subgrid-scale parameterizations. These representations necessarily introduce parameters of which the magnitude is very uncertain.

A typical example is the representation of the horizontal mixing of heat in ocean flows. So-called meso-scale eddies, with typical spatial scales of $10-50 \mathrm{~km}$, take care of much of this mixing. In ocean models with a too coarse horizontal resolution, say $1^{\circ}$, the effect of these eddies cannot be adequately captured and the net horizontal heat flux $\Phi$ is very often approximated as

$\Phi=-K_{H} \nabla_{H} T$,

where $T$ is the temperature and $K_{H}$ is a so-called eddy diffusivity. Similar parameterizations are used for the transport of momentum and in this case the coefficients are referred to

Correspondence to: A. D. Terwisscha van Scheltinga

(a.d.terwisschavanscheltinga@ phys.uu.nl) as eddy-viscosities. If $A_{H}$ is the horizontal eddy viscosity in a coarse resolution model, then estimates of $A_{H}$ range from $10^{3} \mathrm{~m}^{2} \mathrm{~s}^{-1}$ to $10^{5} \mathrm{~m}^{2} \mathrm{~s}^{-1}$. In a flow having a typical length scale $L$ and a horizontal velocity scale $U$, the Reynolds number

$R e=\frac{U L}{A_{H}}$

is hence a very uncertain parameter.

The parameterization of subgrid-scale processes introduces model errors and one cannot expect that the large-scale ocean flows simulated resemble the ones observed. The quality of these simulations can, however, be substantially improved by using observations in a data-assimilation framework. Within this framework, the parameter estimation procedure is aimed at choosing an optimal parameter vector in an admissible parameter volume, so that the model solution corresponding to this parameter vector is "close to" observations.

One of the data-assimilation approaches used is the ensemble Kalman filter method (EnKF), which is an efficient Monto-Carlo approximation to optimal Kalman filtering (Kalman, 1960; Evensen, 1994, 2003). Although this method is generally used for initial state estimation, Derber (1989); Anderson (2001); Hargreaves et al. (2004) suggested the application of EnKF for parameter estimation, by considering the parameters as additional state variables. This method was recently applied by Annan and Hargreaves (2004) to estimate a single parameter in the Lorenz (1963) model. In Annan et al. (2005), the method was applied to estimate parameters in an intermediate-complexity climate model.

A second approach used is variational data assimilation with 4D-Var as a typical method. In this method, all information that is present in observations is combined with the evolution determined by a particular ocean, atmosphere or climate model. In the $4 \mathrm{D}$-Var approach, a cost function is minimized by varying the initial condition and/or the forcing of the model. This cost function measures the distance 
between the data and a state vector at a sequence of times. The so-called analysis is that state which minimizes the cost function and the minimization procedure requires the evaluation of the gradient of the cost function. The $4 \mathrm{D}$-Var method is routinely applied at ECMWF in weather forecasting (Rabier et al., 2000; Mahfouf and Rabier, 2000; Klinker et al., 2000). The method is also used in operational oceanography, for example within the French Mercator project (Weaver et al., 2003; Vialard et al., 2003) where the use of observations to initialize ocean circulation models results in better forecasts.

Parameter estimation using variational methods has been used for example by Yu and O'Brien (1991) to estimate wind-stress coefficients and eddy-viscosity profiles. Zhu and Navon (1999) study adjustment of three parameters, one of them being a horizontal eddy viscosity, in the Florida State University global atmosphere model using a variational approach. They combine 4D-Var with a penalty function method to transform the constrained optimization problem into an unconstrained optimization problem. They show that maximum benefit is obtained from the combined effect of both parameter estimation and initial condition optimization. An overview of many of the current parameter estimation methods used is presented in Navon (1998).

In general, the gradient of the cost function in the 4DVar method is calculated by using both the forward and the adjoint model. In this paper, we show that when a fullyimplicit model is available, 4D-Var can be performed without the need for an explicit adjoint model. The gradient can be computed by using the transpose of the Jacobian matrix that is available during the implicit time stepping. This implicit variant of 4D-Var, called I4D-Var, is highly suitable for strongly nonlinear problems, since the Jacobian (the tangent linear model) is evaluated at each time step and hence varies over a single assimilation interval. In addition, we show how I4D-Var can be adapted for parameter estimation. The capabilities of the resulting method are shown for the barotropic quasi-geostrophic model of the double-gyre winddriven ocean circulation. From the bifurcation diagrams for these flows (Dijkstra and Katsman, 1997), the different flow regimes (steady, periodic and quasi-periodic) are known and hence the parameter estimation problem can be studied systematically. Using synthetic observations from the same model, we will show that I4D-Var is able to correctly estimate parameter values, even when background flow and (synthetic) observations are in different dynamical regimes.

\section{The I4D-Var method}

To describe the version of 4D-Var for implicit models and its extension for multi-parameter estimation, we start by summarizing the main steps of 4D-Var.

\subsection{A summary of 4D-Var}

Let $\boldsymbol{w}$ be the state vector consisting of model variables that are to be estimated by combining model dynamics and observations. If $\boldsymbol{w}^{b}$ is the background state and $\delta \boldsymbol{w}$ is the increment on the background state, then with $4 \mathrm{D}$-Var one wants to determine $\delta \boldsymbol{w}$ such that the resulting state $\boldsymbol{w}$ defined by

$\boldsymbol{w}=\boldsymbol{w}^{b}+\delta \boldsymbol{w}$.

is "close" to observations. Let $M=M\left(t_{i}, t_{i-1}\right)$ represent the evolution operator of the particular model used, such that

$\boldsymbol{w}\left(t_{i}\right)=M\left(t_{i}, t_{i-1}\right)\left(\boldsymbol{w}\left(t_{i-1}\right)\right)$.

Substitution of Eq. (3) into Eq. (4) and linearizing around $\boldsymbol{w}^{b}\left(t_{i}\right)$ gives:

$\boldsymbol{w}\left(t_{i}\right) \approx M\left(t_{i}, t_{i-1}\right)\left(\boldsymbol{w}^{b}\left(t_{i-1}\right)\right)+\mathbf{M}\left(t_{i}, t_{i-1}\right) \delta \boldsymbol{w}\left(t_{i-1}\right)$,

where $\mathbf{M}\left(t_{i}, t_{i-1}\right)$ is the tangent linear operator,

$\left.\mathbf{M} \equiv \frac{\partial M}{\partial \boldsymbol{w}}\right|_{\boldsymbol{w}=\boldsymbol{w}^{b}}$,

and $\delta \boldsymbol{w}\left(t_{i}\right)=\mathbf{M}\left(t_{i}, t_{i-1}\right) \delta \boldsymbol{w}\left(t_{i-1}\right)$ is the corresponding tangent-linear model. Let $\boldsymbol{y}_{i}$ denote the vector of observations and $H_{i}$ the observation operator at time $t_{i}$, then

$\boldsymbol{y}_{i}=H_{i}\left(\boldsymbol{w}\left(t_{i}\right)\right) \approx H_{i}\left(\boldsymbol{w}^{b}\left(t_{i}\right)\right)+\mathbf{H}_{i} \delta \boldsymbol{w}\left(t_{i}\right)$,

where $\mathbf{H}_{i}$ is the linearization of $H_{i}$ around the background state. By the hypothesis of causality, we have

$M\left(t_{i}, t_{0}\right)=M\left(t_{i}, t_{i-1}\right) \cdots M\left(t_{1}, t_{0}\right)$,

$\mathbf{M}\left(t_{i}, t_{0}\right)=\mathbf{M}\left(t_{i}, t_{i-1}\right) \cdots \mathbf{M}\left(t_{1}, t_{0}\right)$,

and the model estimates of the observations can be linked to the initial conditions at $t=t_{0}$ through Eq. (8a) as

$H_{i}\left(\boldsymbol{w}\left(t_{i}\right)\right) \approx H_{i} M\left(t_{i}, t_{0}\right)\left(\boldsymbol{w}^{b}\left(t_{0}\right)\right)+\mathbf{H}_{i} \mathbf{M}\left(t_{i}, t_{0}\right) \delta \mathbf{w}\left(t_{0}\right)$.

In variational methods, such as $4 \mathrm{D}-\mathrm{Var}$, the analysis $\boldsymbol{w}^{a}$ is defined as the state vector which minimizes both the distance to the background $\boldsymbol{w}^{b}\left(t_{0}\right)$ and to the time-sequence of observations $\boldsymbol{y}_{i}$ in the interval $t_{0} \leq t_{i} \leq t_{n}$. If the analysis is close to the background state then the cost function can be written as Courtier et al. (1994):

$J(\delta \boldsymbol{w})=\delta \boldsymbol{w}^{\mathrm{T}} \mathbf{B}^{-1} \delta \boldsymbol{w}+\sum_{i=0}^{n} \boldsymbol{d}_{i}^{T} \mathbf{R}_{i}^{-1} \boldsymbol{d}_{i}$,

where $\mathbf{B}$ is the matrix of background error covariances, $\mathbf{R}_{i}$ is the matrix of observation error covariances, $\delta \boldsymbol{w}$ is the increment on the background state and $n$ is the length of the assimilation intervals (with $n+1$ points). The departures $\boldsymbol{d}_{i}$ are defined as:

$\boldsymbol{d}_{i}=\boldsymbol{y}_{i}-H_{i} M\left(t_{i}, t_{0}\right)\left(\boldsymbol{w}^{b}\left(t_{0}\right)\right)-\mathbf{H}_{i} \mathbf{M}\left(t_{i}, t_{0}\right) \delta \boldsymbol{w}\left(t_{0}\right)$. 
If $\delta \boldsymbol{w}^{a}$ is defined as the solution of the minimization problem, i.e.

$J\left(\delta \boldsymbol{w}^{a}\right)=\min _{\delta \boldsymbol{w}} J(\delta \boldsymbol{w})$

then the analysis at $t_{i}$ is given by

$\boldsymbol{w}^{a}\left(t_{i}\right)=M\left(t_{i}, t_{0}\right)\left(\boldsymbol{w}^{b}\left(t_{0}\right)+\delta \boldsymbol{w}^{a}\right)$,

and the background $\boldsymbol{w}^{b}\left(t_{n+1}\right)$ at the beginning of the next interval is given by:

$\boldsymbol{w}^{b}\left(t_{n+1}\right)=M\left(t_{n+1}, t_{0}\right)\left(\boldsymbol{w}^{b}\left(t_{0}\right)+\delta \boldsymbol{w}^{a}\right)$.

To solve the minimization problem (Eq. 12), the gradient

$\nabla J(\delta \boldsymbol{w})=2 \mathbf{B}^{-1} \delta \boldsymbol{w}-2 \sum_{\mathrm{i}=0}^{\mathrm{n}} \mathbf{M}^{T}\left(t_{i}, t_{0}\right) \mathbf{H}_{i}^{T} \mathbf{R}_{i}^{-1} \boldsymbol{d}_{i}$,

has to be calculated. In an explicit time-stepping ocean, atmosphere or climate numerical model, the usual procedure is to compute this gradient using a forward evolution over the assimilation interval and a backward evolution using the adjoint model, with evolution $\mathbf{M}^{T}\left(t_{i}, t_{i-1}\right)$ and forcing $\mathbf{H}_{i}^{T} \boldsymbol{d}_{i}$. It requires a discrete adjoint model that is well-defined and as efficient as the forward model.

\subsection{D-var for implicit models (I4D-var)}

For models in which implicit time stepping is used, such as a Crank-Nicholson method, no explicit adjoint model is needed. To see why, we first write a model in general operator form as

$\mathcal{T} \frac{\partial \boldsymbol{w}}{\partial t}+\mathcal{L} \boldsymbol{w}+\mathcal{N}(\boldsymbol{w}) \boldsymbol{w}=\mathcal{F}$

where $\mathcal{T}$ and $\mathcal{L}$ are linear operators, $\mathcal{N}$ is a nonlinear operator and $\mathcal{F}$ contains the explicitly known part of forcing. Spatial discretization gives

$\mathbf{T} \frac{\partial \boldsymbol{w}}{\partial t}+\mathbf{L} \boldsymbol{w}+\mathbf{N}(\boldsymbol{w}) \boldsymbol{w}=\boldsymbol{F}$

with T, L, N and $\boldsymbol{F}$ being discretized versions of $\mathcal{T}, \mathcal{L}, \mathcal{N}$ and $\mathcal{F}$, respectively. Using a time step $\Delta t$ with time index $i$, a general implicit scheme can be defined for $\omega \in(0,1]$ as,

$\frac{1}{\Delta t} \mathbf{T}\left(\boldsymbol{w}^{i+1}-\boldsymbol{w}^{i}\right)+(1-\omega)\left(\mathbf{L}+\mathbf{N}\left(\boldsymbol{w}^{i}\right)\right) \boldsymbol{w}^{i}+$
$\omega\left(\mathbf{L}+\mathbf{N}\left(\boldsymbol{w}^{i+1}\right)\right) \boldsymbol{w}^{i+1}=(1-\omega) \boldsymbol{F}^{i}+\omega \boldsymbol{F}^{i+1}$.

For example, for $\omega=1$ the backward Euler method is obtained and for $\omega=1 / 2$ the Crank-Nicholson method. Using the notation $\mathbf{N}^{i}=\mathbf{N}\left(\boldsymbol{w}^{i}\right)$, then re-arranging Eq. (18) gives:

$\left[\frac{1}{\Delta t} \mathbf{T}+\omega\left(\mathbf{L}+\mathbf{N}^{i+1}\right)\right] \boldsymbol{w}^{i+1}=\boldsymbol{G}^{i}$

$\boldsymbol{G}^{i}=\left[\frac{1}{\Delta t} \mathbf{T}-(1-\omega)\left(\mathbf{L}+\mathbf{N}^{i}\right)\right] \boldsymbol{w}^{i}+(1-\omega) \boldsymbol{F}^{i}+\omega \boldsymbol{F}^{i+1}$.
This nonlinear system of equations is solved using the Newton-Raphson method. Let the Newton iteration index be indicated by $l$ and $\mathbf{N}^{i+1, l}$ be the linearization of $\mathbf{N}^{i+1}$ around $\boldsymbol{w}^{i+1, l}$. For the system (Eq. 19a), the Newton-Raphson method is:

$$
\begin{aligned}
& \boldsymbol{w}^{i+1,0}=\boldsymbol{w}^{i}, \\
& \boldsymbol{w}^{i+1, l+1}=\boldsymbol{w}^{i+1, l}+\Delta \boldsymbol{w}^{i+1, l+1}, \\
& \mathbf{J} \Delta \boldsymbol{w}^{i+1, l+1}=\mathbf{J} \boldsymbol{w}^{i+1, l}+\boldsymbol{G}^{i}, \\
& \mathbf{J}=\frac{1}{\Delta t} \mathbf{T}+\omega\left(\mathbf{L}+\mathbf{N}^{i+1, l}\right) .
\end{aligned}
$$

and the linear system (Eq. 20c) has to be solved for each iteration. The relation (Eq. 18) provides an explicit representation of the spatially discretized evolution operator as:

$M\left(t_{i+1}, t_{i}\right)\left(\boldsymbol{w}\left(t_{i}\right)\right)=\left[\frac{1}{\Delta t} \mathbf{T}+\omega\left(\mathbf{L}+\mathbf{N}^{i+1}\right)\right]^{-1} \boldsymbol{G}_{i}$

The spatially discretized tangent linear model follows from linearization of this operator around $\boldsymbol{w}^{b}\left(t_{i}\right)$ and becomes

$$
\begin{aligned}
\left.\mathbf{M} \equiv \frac{\partial M}{\partial \boldsymbol{w}}\right|_{\boldsymbol{w}=\boldsymbol{w}^{b}}= & {[\underbrace{[\underbrace{\frac{1}{\Delta t} \mathbf{T}+\omega\left(\mathbf{L}+\mathbf{N}^{i+1}\right)}_{\mathbf{C}_{2, i}}]^{-1}}_{\mathbf{C}_{1, i}}} \\
& \underbrace{\left[\frac{1}{\Delta t} \mathbf{T}-(1-\omega)\left(\mathbf{L}+\mathbf{N}^{i}\right)\right]}
\end{aligned}
$$

and it can be explicitly written as

$\mathbf{M}\left(t_{i+1}, t_{i}\right)=\mathbf{C}_{1, i}^{-1} \mathbf{C}_{2, i}$.

As the Jacobian matrix $\mathbf{J}$ is available during the NewtonRaphson iteration, one gets the tangent-linear model and its transpose, to be used in the computation of the cost function in 4D-Var, nearly for free. This approach has another advantage: the tangent linear model is adapted at each time step and hence I4D-Var is expected to perform better in strongly nonlinear problems than the original 4D-Var method.

\subsection{Parameter estimation}

As mentioned in the introduction, typically parameter values are uncertain in ocean, atmosphere or climate models, in particular those associated with the mixing of, for example, heat and momentum. The typical problem which we consider here is one in which the parameters guessed are far from the value needed for the model solution to be close to observational values. When parameters are not adapted, 4D-Var may improve the results of badly tuned models but usually large error will remain. How can one adapt 4D-Var to change these parameters to "correct" values during assimilation? 
Parameter estimation is difficult in 4D-Var, since a change in the underlying vector field due to a parameter variation cannot be easily taken into account. In I4D-Var, however, the parameter dependence of the local Jacobian matrix is explicitly available. Let $\boldsymbol{p}$ be the vector of parameters and rewrite the cost function (10) to explicitly include the parameters in its formulation, i.e.

$J(\delta \boldsymbol{w}, \boldsymbol{p})=\sum_{i=0}^{n} \boldsymbol{d}_{i}^{T} \mathbf{R}_{i}^{-1} \boldsymbol{d}_{i}$,

where the departures $\boldsymbol{d}_{i}$ are given by

$\boldsymbol{d}_{i}=\boldsymbol{y}_{i}-H_{i} M\left(t_{i}, t_{0}, \boldsymbol{p}\right)\left(\boldsymbol{w}^{b}\left(t_{0}\right)\right)-\mathbf{H}_{i} \mathbf{M}\left(t_{i}, t_{0}, \boldsymbol{p}\right) \delta \boldsymbol{w}$.

and $M\left(t_{i}, t_{0}, \boldsymbol{p}\right)$ represents the evolution operator. The minimization problem now becomes:

$\min _{\delta \boldsymbol{w}, \boldsymbol{p}} J(\delta \boldsymbol{w}, \boldsymbol{p})$.

When a simultaneous minimization is attempted over both the initial condition or forcing and the parameters, the cost function is no longer quadratic since the introduction of the parameters as control variables gives additional nonlinearities. Hence, a unique minimum is no longer guaranteed; a different approach is needed.

In Zhu and Navon (1999), the cost function is extended by including a penalty term $\lambda^{T} \boldsymbol{g}(\boldsymbol{p})$, where the penalty coefficient vector $\lambda$ is determined such that penalty term is of the same order as the other terms in the cost function. The quadratic vector function $\boldsymbol{g}(\boldsymbol{p})$ is introduced to set the boundaries in the parameter space. The advantage is that the cost function is again quadratic, but the direct disadvantage is that the results of the analysis can be very sensitive to the specification of the penalty coefficient vector (Nash and Sofer, 1996).

In I4D-Var, the Jacobian matrix is explicitly available at each time step, while the derivative of the vector field to each parameter can be made available. Hence, instead of simultaneously minimizing over $\delta \boldsymbol{w}$ and $\boldsymbol{p}$, one can attempt to minimize sequentially over $\delta \boldsymbol{w}$ and $\boldsymbol{p}$. In this approach, we first determine $\delta \boldsymbol{w}^{a}$ as a solution of the minimization problem

$$
\min _{\delta \boldsymbol{w}} J\left(\delta \boldsymbol{w}, \boldsymbol{p}^{b}\right)
$$

with $\delta \boldsymbol{w}=\mathbf{0}$ as a first guess for the minimization and $\boldsymbol{p}^{b}$ is the parameter vector for which the background has been calculated. This minimization problem yields an analysis at $t_{i}$ given by

$\boldsymbol{w}^{a}\left(t_{i}\right)=M\left(t_{i}, t_{0}, \boldsymbol{p}^{b}\right)\left(\boldsymbol{w}^{b}\left(t_{0}\right)+\delta \boldsymbol{w}^{\mathrm{a}}\right)$.

Next, we determine $\boldsymbol{p}^{a}$ such that the analysis (Eq. 28) is improved. This can be done by minimizing

$\min _{\boldsymbol{p}} J\left(\delta \boldsymbol{w}^{a}, \boldsymbol{p}\right)$,

where the linearization around the background state has been dropped, i.e. the departures are taken as

$\boldsymbol{d}_{i}=\boldsymbol{y}_{i}-H_{i} M\left(t_{i}, t_{0}, \boldsymbol{p}\right)\left(\boldsymbol{w}^{b}\left(t_{0}\right)+\delta \boldsymbol{p}\right)$.
As a first guess, the parameters of the background are taken as $\boldsymbol{p}=\boldsymbol{p}^{b}$. When these problems are solved, then the analysis is found from

$\boldsymbol{w}^{a}\left(t_{i}\right)=M\left(t_{i}, t_{0}, \boldsymbol{p}^{\mathrm{a}}\right)\left(\boldsymbol{w}^{b}\left(t_{0}\right)+\delta \boldsymbol{w}^{a}\right)$,

and the background $\boldsymbol{w}^{b}\left(t_{n+1}\right)$ at the beginning of the next interval is given by:

$\boldsymbol{w}^{b}\left(t_{n+1}\right)=M\left(t_{n+1}, t_{0}, \boldsymbol{p}^{a}\right)\left(\boldsymbol{w}^{b}\left(t_{0}\right)+\delta \boldsymbol{w}^{a}\right)$.

This sequential minimization has several advantages over Eq. (26). First, for the minimization over $\delta w$ in Eq. (27), the cost function remains quadratic and hence a unique minimum can be expected. Secondly, minimizing over the initial conditions first, yields an improvement of the model solution. This improvement gives an indication whether the current estimate $\boldsymbol{p}^{b}$ is accurate. If not, the initial condition $\delta \boldsymbol{w}^{a}$ gives an analysis $\boldsymbol{w}^{a}\left(t_{0}\right)$, which is close to the observation $\boldsymbol{y}_{0}$. Fixing $\delta \boldsymbol{w}=\delta \boldsymbol{w}^{a}$ introduces a strong constraint on the minimization problem (Eq. 29). Though $J(\delta \boldsymbol{w}, \boldsymbol{p})$ is non-linear and therefore multiple minima of Eq. (29) may be expected, this constraint reduces the number of feasible minima. As a result, the computation is numerically better conditioned. The main advantage of I4D-Var over parameter estimation with 4D-Var is that I4D-Var takes in account changes in the state due to a parameter variation, since the Jacobian is evaluated for each time step and therefore also the parameter dependence of the local Jacobian.

To test the I4D-Var method obtained in this way, one would like a problem for which it is known that different parameter values lead to a qualitatively different type of flow behavior. For such a problem, parameters can be chosen in one flow regime (for example, a regime where only one steady state solution exists for $t \rightarrow \infty$ ) whereas synthetic observations can be chosen at parameter values in another flow regime (for example, a regime of multiple steady states, or (quasi-) periodic behavior). The example below of the winddriven circulation in an idealized ocean basin is ideally suited as such a problem, since the regime boundaries have been studied extensively (Dijkstra and Katsman, 1997).

\section{The quasi-geostrophic barotropic double-gyre flow}

We consider a rectangular ocean basin of size $L \times L$ having a constant depth $D$. The basin is situated on a midlatitude $\beta$ plane with a central latitude $\theta_{0}=45^{\circ} \mathrm{N}$ and Coriolis parameter $f_{0}=2 \Omega \sin \theta_{0}$, where $\Omega$ is the rotation rate of the Earth. The variation of the Coriolis parameter at the latitude $\theta_{0}$ is indicated by $\beta_{0}$. The density $\rho$ of the water is constant and the flow is forced at the surface through a wind-stress vector $\tau_{0}\left[\tau^{x}(x, y), \tau^{y}(x, y)\right]$. The governing equations are nondimensionalized using the horizontal length scale $L$, the vertical length scale $D$, a horizontal velocity scale $U$ and the advective time scale $L / U$. A typical choice of the horizontal velocity scale $U$ is based on the Sverdrup balance and is given by

$U=\frac{\tau_{0}}{\rho D \beta_{0} L}$. 
The effect of ocean-atmosphere deformations on the flow is neglected. The dimensionless barotropic quasigeostrophic model of the flow for the vorticity $\zeta$ and the geostrophic streamfunction $\psi$ is (Pedlosky, 1987)

$\left[\frac{\partial}{\partial t}+u \frac{\partial}{\partial x}+v \frac{\partial}{\partial y}\right][\zeta+\beta y]=R e^{-1} \nabla^{2} \zeta$

$+\alpha_{\tau}\left(\frac{\partial \tau^{y}}{\partial x}-\frac{\partial \tau^{x}}{\partial y}\right)$

$\zeta=\nabla^{2} \psi$

where the horizontal velocities are given by $u=-\partial \psi / \partial y$ and $v=\partial \psi / \partial x$. This equation contains several parameters. These are the Reynolds number $R e$, the planetary vorticity gradient parameter $\beta$ and the wind-stress forcing strength $\alpha_{\tau}$. These parameters are defined as:

$R e=\frac{U L}{A_{H}} ; \quad \beta=\frac{\beta_{0} L^{2}}{U} ; \quad \alpha_{\tau}=\frac{\tau_{0} L}{\rho D U^{2}}$

where $g$ is the gravitational acceleration and $A_{H}$ is the lateral friction coefficient. If the characteristic velocity $U$ is chosen as in Eq. (22), it follows that $\alpha_{\tau}=\beta$ and there are only two independent parameters in the problem. We assume no-slip conditions on the east-west boundaries and slip conditions on the north-south boundaries. The boundary conditions are therefore given by

$x=0, x=1: \psi=\frac{\partial \psi}{\partial x}=0$,

$y=0, y=1: \psi=\zeta=0$.

The wind-stress forcing is prescribed as

$\tau^{x}(x, y)=\frac{-1}{2 \pi}((1-a) \cos (2 \pi y)+a \cos (\pi y))$,

$\tau^{y}(x, y)=0$.

with $a$ being an additional dimensionless parameter controlling the symmetry of the zonal wind stress. For $a=0$ the wind stress is symmetric, with easterlies at the northern and southern boundaries of the domain and westerlies at the midaxis of the basin.

The governing equations were discretized on a equidistant $N \times M$ grid using central spatial differences. The CrankNicholson scheme was used in the time-integration, the nonlinear system of algebraic equations was solved with the Newton-Raphson method and the emerging linear systems were solved iteratively with a preconditioned conjugate gradient method. The gradient Eq. (15) was calculated using backward iteration, which required the transposition of Eq. (23) and one extra linear system to be solved per iteration. The derivative of $J$ with respect to a parameter $p_{j}$ was, when possible, calculated by differentiation of the discretized equations Eq. (18) with respect to $p_{j}$. Otherwise finite differences were used according to

$$
\left.\frac{\partial J}{\partial \boldsymbol{p}_{j}}\right|_{\boldsymbol{p}=\boldsymbol{p}^{*}} \approx \frac{J\left(\delta \boldsymbol{w}, \boldsymbol{p}^{*}+\varepsilon \boldsymbol{e}_{j}\right)-J\left(\delta \boldsymbol{w}, \boldsymbol{p}^{*}\right)}{\varepsilon},
$$

Table 1. Standard values of the parameters for the barotropic quasigeostrophic ocean model in the steady flow regime.

\begin{tabular}{lll}
\hline Parameter & Value & \\
\hline$L$ & $1.0 \times 10^{6}$ & $\mathrm{~m}$ \\
$U$ & $7.1 \times 10^{-3}$ & $\mathrm{~m}$ \\
$D$ & $7.0 \times 10^{2}$ & $\mathrm{~m}$ \\
$\beta_{0}$ & $2.010^{-11}$ & $(\mathrm{~ms})^{-1}$ \\
$f_{0}$ & $1.0 \times 10^{-4}$ & $\mathrm{~s}^{-1}$ \\
$g$ & 9.8 & $\mathrm{~ms}^{-2}$ \\
$\rho$ & $1.0 \times 10^{3}$ & $\mathrm{kgm}^{-3}$ \\
$\tau_{0}$ & $1.0 \times 10^{-1}$ & $\mathrm{~Pa}^{-1}$ \\
\hline Parameter & Value & \\
$\alpha_{\tau}=\beta$ & $2.8 \times 10^{3}$ & \\
$a$ & 0.0 & \\
\hline
\end{tabular}

where $\boldsymbol{e}_{j}$ is the j-th unit vector, $\boldsymbol{p}^{*}$ is the point at which the gradient of $J$ with respect to $p$ is evaluated and $\varepsilon$ small. The evaluation of the gradient with respect to one parameter requires two evaluations of the cost function $J$ and in comparison with the gradient of the cost function with respect to $\delta \boldsymbol{w}$ does not require storage of the Jacobian, nor backward iteration.

\section{Results}

In this section, we will show the performance of I4D-Var on three test problems using the barotropic ocean model as described in the previous section. The latter model is used as the background model during assimilation and parameter estimation and also for generation of the "observations" of the streamfunction $\psi$. A standard set of parameter values was chosen that are similar to those in Dijkstra and Katsman (1997) and these values are given in Table 1. With the choice of $U$ as in Eq. (33) and the wind stress as in Eq. (37a), there are three independent dimensionless parameters in the system. We fix the value of $\beta$ and consider $R e, \alpha_{\tau}$ and $a$ as our "uncertain" parameters.

For the parameters as in Table 1, Dijkstra and Katsman (1997) showed that there are several flow regimes depending on the value of $R e$. For $R e<30$ the background model has one unique steady-state symmetric (with respect to the basin's mid-axis) double-gyre solution. For $30<R e<52$, two stable asymmetric steady-state solutions exist, one with a northward jet displacement (the so-called jet-up solution) and one with a southward jet displacement (the jet-down solution). Both asymmetric steady states become unstable for $R e>52$ due to the occurrence of Hopf bifurcations. For $52<R e<74$ periodic orbits exists, while for $R e>74$ the solutions are first quasi-periodic and thereafter become very irregular. The boundaries between these qualitatively different dynamical regimes depend on the values of $\alpha_{\tau}$ and $a$. For a nonzero value of $a$, the reflection symmetry with respect to 
Table 2. The values of the dimensionless parameter for each of the cases I, ..., VI considered.

\begin{tabular}{llll}
\hline Regime & $\alpha_{\tau}$ & $R e$ & $a$ \\
\hline I & 2800 & 20 & 0.0 \\
II & 2800 & 50 & 0.0 \\
III & 2800 & 120 & 0.0 \\
IV & 2200 & 20 & -0.2 \\
V & 3400 & 50 & 0.2 \\
VI & 3400 & 120 & -0.2 \\
\hline
\end{tabular}

the mid-axis of the basin is broken and no symmetric double gyre solutions exist anymore.

All solutions below are calculated with a time-step of 1 day on an equidistant $60 \times 40$ grid. For moderate values of the Reynolds number $R e$, this resolution is sufficient to capture an accurate representation of the solutions (Dijkstra and Katsman, 1997). Six different parameter sets were considered to illustrate the capabilities of the I4D-Var method (see Table 2). To show the behavior of the background model in each of these cases, the time evolution of the basin integrated kinetic energy is shown in Fig. 1. For case I, for which $a=0$, a steady symmetric state is obtained of which the streamfunction $\psi$ is plotted in Fig. 2a. At a slightly larger value of $R e=50$ (case II), an asymmetric steady state (Fig. 2b) is obtained which is a "jet-up" solution. For an even larger value of $R e=120$ (case III), the flow is time-dependent and the time-mean of the streamfunction averaged over a 4000 day period is shown in Fig. 2c. For the cases IV, V and $\mathrm{VI}$, the wind-stress forcing is asymmetric $(a \neq 0)$. While the flows for the cases IV and V approach steady states (shown in Fig. 2d and Fig. 2e, respectively), the flow for case VI is again time-dependent and the time-mean state is plotted in Fig. 2f.

The steady state and time-dependent streamfunction fields were used as the initial background or as synthetic observations in the data-assimilation runs presented below. The observations of $\psi$ at all the gridpoints were used, i.e. $H_{i}$ is equal to the identity operator for all $i$. Two types of test problems were considered: single-parameter and multi-parameter estimation. For multi-parameter estimation, a total of 50 iterations were calculated, each with one sequential minimization as described in Sect. 2.3, and we use 6 points per assimilation interval. For the single-parameter estimation runs, the computation was terminated after an increase in the optimized cost function was detected at subsequent intervals. For these test problems, 5 point per assimilation interval were used.

\subsection{Single parameter estimation}

In these test-problems, we use $R e$ as the uncertain parameter, while the values of $\alpha_{\tau}$ and $a$ are fixed. As a first test, the unique steady-steady state solution of case I (Fig. 2a) for $R e=20$ is taken as the initial background and the synthetic

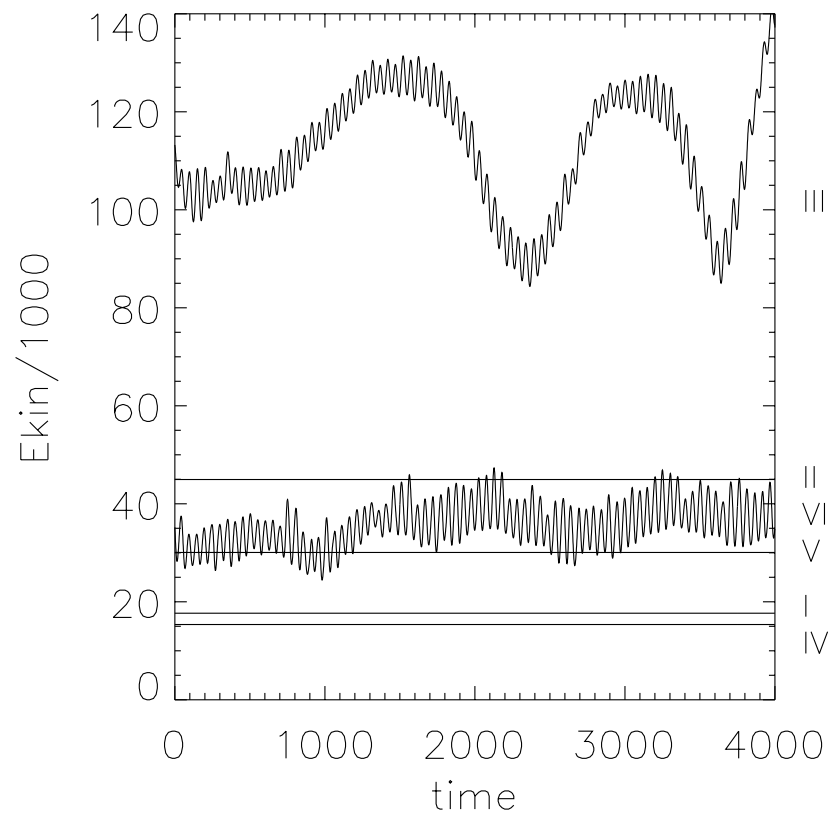

Fig. 1. Time evolution of the basin integrated kinetic energy for the different cases I, ..., VI.

observations are derived from the steady-state "jet-up" solution of case II (Fig. 2b) for $R e=50$.

After a few intervals, the estimate of $R e$ computed with I4D-Var is already close to "correct" value $R e=50$ and eventually it converges toward this value (Fig. 3a). The value of the cost function for each interval - before minimization over the initial conditions (drawn) and after minimization over $R e$ (dashed) - is shown in Fig. 3b. The value of the cost function converges to zero, indicating that a perfect fit to the synthetic observations is found. For the first interval, the value of the cost function is reduced by about three orders of magnitude after minimization over the initial conditions. In the remaining intervals, a decrease of about one order of magnitude is found.

In Fig. 3c, the $L_{2}$-norm of the differences between the observations and the initial background (drawn) and between the observations and the analysis (dashed) are plotted. The difference between analysis and observations is for all intervals smaller than the difference between observations and background and both norms converge to zero indicating that a perfect fit has been found. The difference between the observations and the background increases over the assimilation interval which indicates that the background is still attracted away from the observations. However, this effect decreases when the estimate for $R e$ approaches $R e=50$. After the first interval both differences are close to each other at the first point in the second interval. This shows that the model solution at this point is already close to the observations. This is in agreement with the decrease of three orders of magnitude in the cost function as seen in Fig. $3 b$.

The $L_{2}$-norm of the initial gradient of the cost function with respect to the increment (drawn) before sequential mini- 

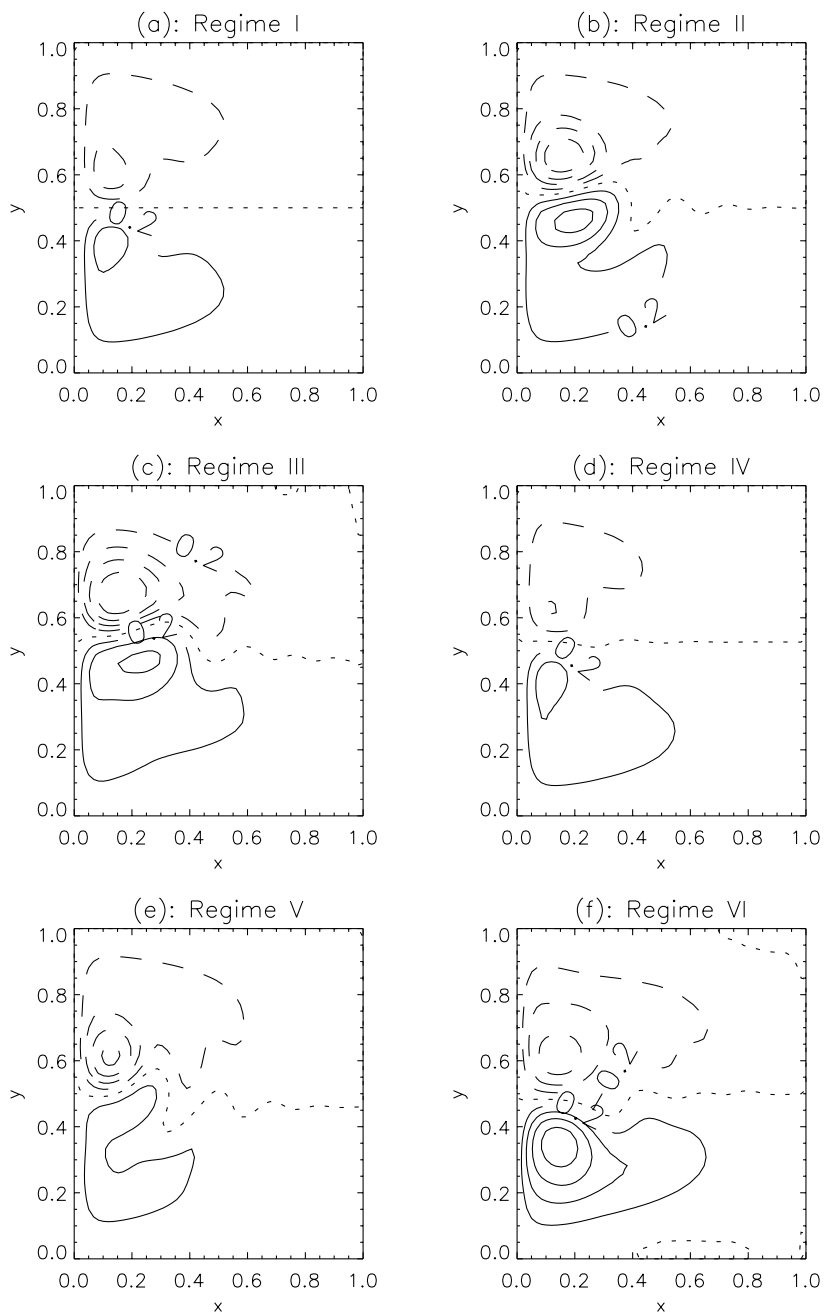

Fig. 2. Contour plots of the streamfunction field: (a) the steady state of case I; (b) a steady state of case II; (c) a time-mean field of case III; (d) steady state of case IV; (e) a steady state of case V; and (f) a time-mean field of case VI. The contours are with respect to a maximum over these 6 fields of $\psi=2.25$, which is equivalent with a transport of $12.4 \mathrm{~Sv}\left(1 \mathrm{~Sv}=10^{6} \mathrm{~m}^{3} \mathrm{~s}^{-1}\right)$.

mization and after minimization over $R e$ (dashed) are plotted in Fig. 3d. The convergence of the gradient to zero indicates again that both a perfect fit to observations and an accurate estimate of $R e$ have been found. During the first 14 intervals, the dashed curve is above the dotted curve (Fig. 3d), but the distance between the curves is decreasing and becomes negligibly small after the 14th interval. This indicates that during the first 14 intervals, (large) improvements in $R e$ and/or the background model are still possible but that after the 14th interval, the state-parameter solution is very close to the solution corresponding to the observations. In summary, for the case in which the initial background and the "observations" are in different dynamical regimes - a unique steady regime (case I) and a multiple equilibria regime (case II) the performance of I4D-Var is very good.
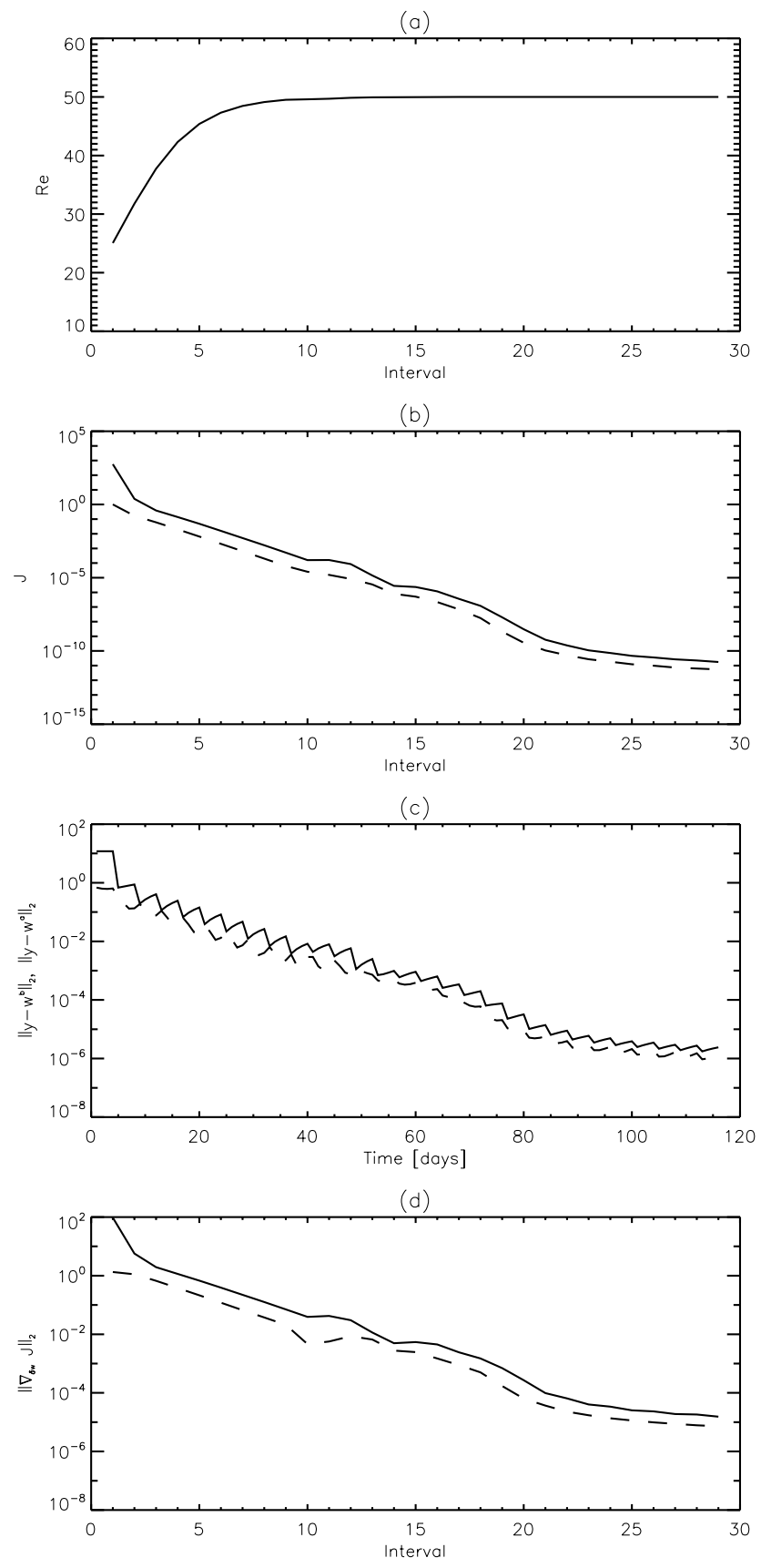

Fig. 3. Results for initial background from case I and observations from case II: (a) $R e$ versus the number of intervals (note that the number of intervals is equal to the number of sequential minimizations of $J$ ); (b) the initial value of the cost function (drawn), its value after minimization over the initial conditions (dotted) and after minimization over $R e$ (dashed); (c) the $L_{2}$-norm of the difference between the observations and the initial background (drawn) and the difference between the observations and the analysis (dashed); (d) the $L_{2}$-norm of the initial gradient of the cost function with respect to the increment (drawn), its value after minimization over the initial conditions (dotted) and after minimization over $R e$ (dashed). 


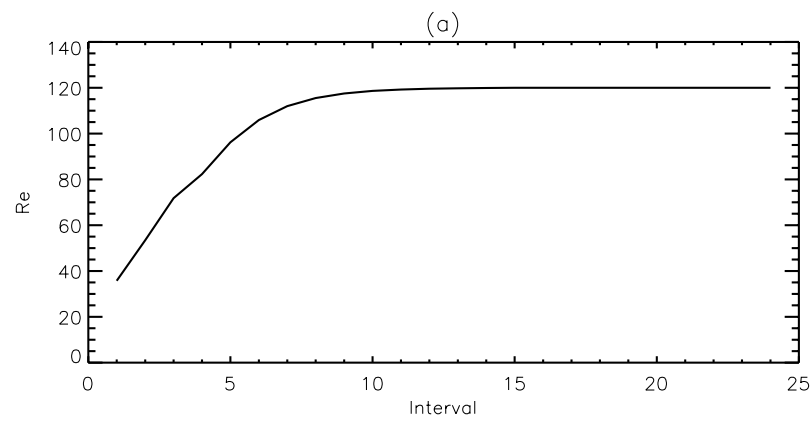

(b)

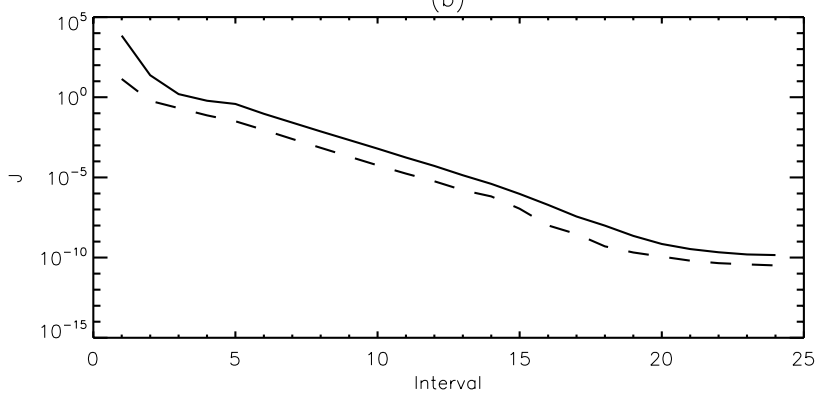

(c)

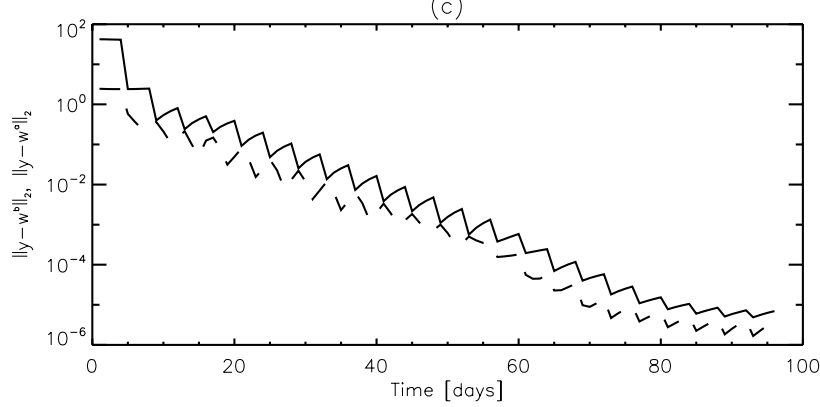

(d)

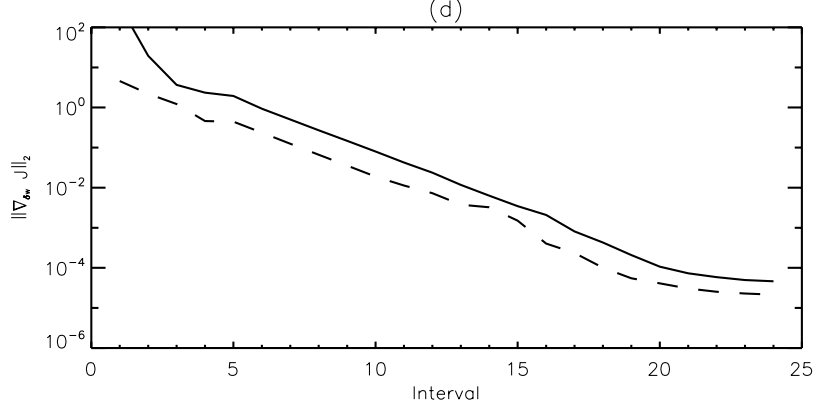

Fig. 4. Results for initial background from case I and observations from case III: (a) $R e$ versus the number of intervals (note that the number of intervals is equal with the number of sequential minimizations of $J$ ); (b) the initial value of the cost function (drawn), its value after minimization over the initial conditions (dotted) and after minimization over $R e$ (dashed); (c) the $L_{2}$-norm of the differences between the observations and the initial background (drawn) and between the observations and the analysis (dashed); (d) the $L_{2}$ norm of the initial gradient of the cost function with respect to the increment (drawn), its value after minimization over the initial conditions (dotted) and after minimization over $R e$ (dashed).

The second problem to test I4D-Var is slightly more complicated as we use the time-dependent observations from case III $(R e=120)$ and as initial background the steady state of case I $(R e=20)$. As the results in Fig. 4 show, I4D-Var is able to estimate the correct value of $\operatorname{Re}$ (Fig. $4 \mathrm{a}$ ) and the convergence of the different norms is similar (Fig. $4 b-d)$ to that in Fig. 3. This indicates that I4D-Var is also capable of efficiently estimate an uncertain parameter for highly transient observations.

Several other test problems, with other combinations of dynamical behavior - i.e. steady state, periodic, quasiperiodic and irregular, for the initial background and observations - were investigated. The I4D-Var method worked equally well for these problems.

\subsection{Multi parameter estimation}

Using the barotropic model of the wind-driven circulation, we can also test the performance of I4D-Var in a multiparameter estimation problem. A maximum of three uncertain parameters, $R e, \alpha_{\tau}$ and $a$, can be considered. In all the test problems below, the initial background is the unique steady state of case IV (Fig. 2d). Due to a negative value of $a$, this steady state has a small southward jet displacement when compared to the symmetric steady-state of case I (Fig. 2a).

In the first problem, the parameters of case $\mathrm{V}$ are estimated by taking its steady-state (Fig. 2e) as the observations. Note that case $\mathrm{V}$ has different values for all three parameter than case IV and that, in particular, the value of $a$ has opposite sign. The steady state in case $\mathrm{V}$ is a jet-up solution and hence substantially different than that of case IV (Fig. 2d). Due to the higher value of $\alpha_{\tau}$ and $R e$, the amplitude of the flow is also a lot stronger.

The I4D-Var method is able to find accurate estimates for all parameters. After 10 intervals the estimated values of all three parameters are close to those of case V (Fig. 5ac). In Fig. 5d, the final value of the cost function after minimization is one order of magnitude smaller than its value before sequential minimization for all intervals, and two orders of magnitude smaller for the first interval. During the first twenty intervals, both values of the cost function rapidly decrease, but after 22 intervals convergence is much slower. This is due to the minimization routine used, which terminates when the difference of the cost function $J$ between successive iterates is smaller than $10^{-5}$. After 22 intervals, the initial value of the cost function before minimization over the parameters is smaller than the stop criteria. As a result, the minimization routine will always terminate after one iteration, which leads to less improvement of the cost function and decrease convergence. This has no consequences for the result. After 20 intervals the final values of the cost function is of order $10^{-4}$, and hence the analysis is already sufficiently close to the observations. Furthermore, the estimates for the parameter are accurate after 20 intervals.

For this, and the following test problems, we do not show anymore the differences between the observations and initial background and between observations and analysis, nor the gradient of the cost function with respect to the increment because these figures look qualitatively the same as Fig. 3c,d and Fig. 4c,d. The results show that I4D-Var is capable of 
(a)

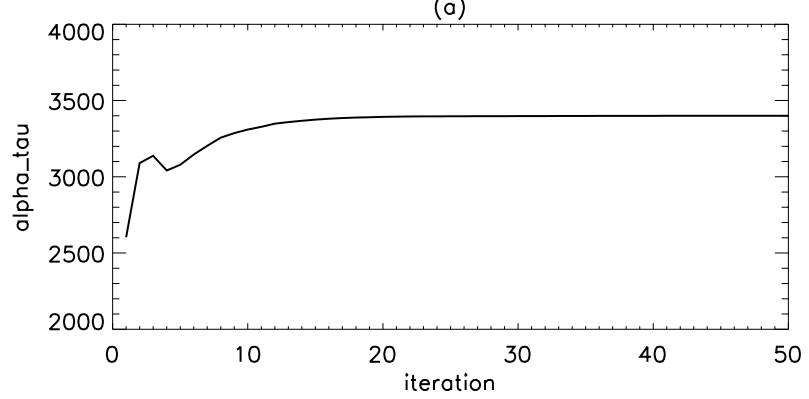

(b)

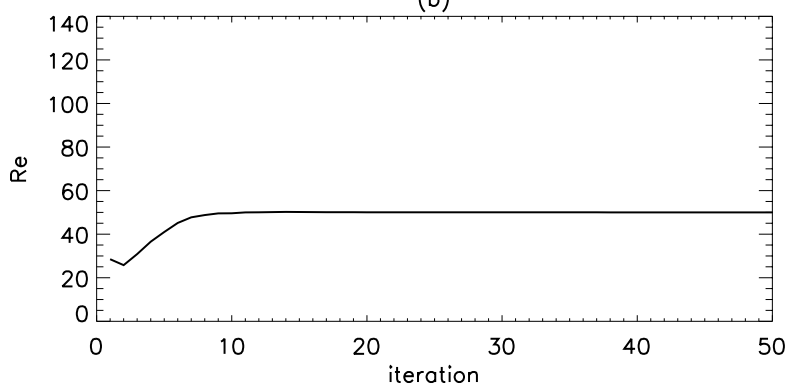

(c)

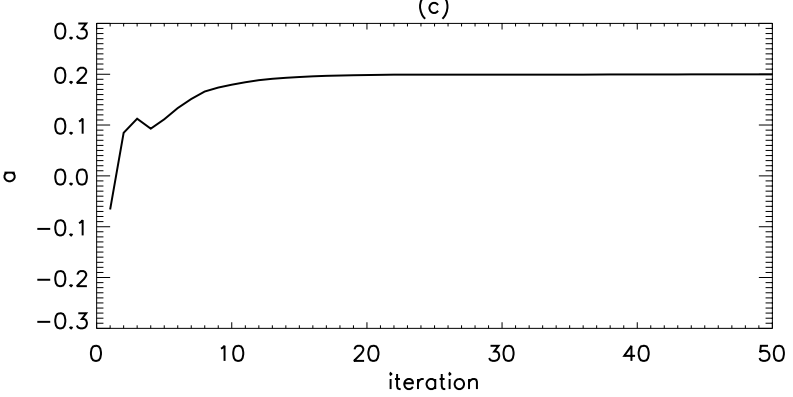

(d)

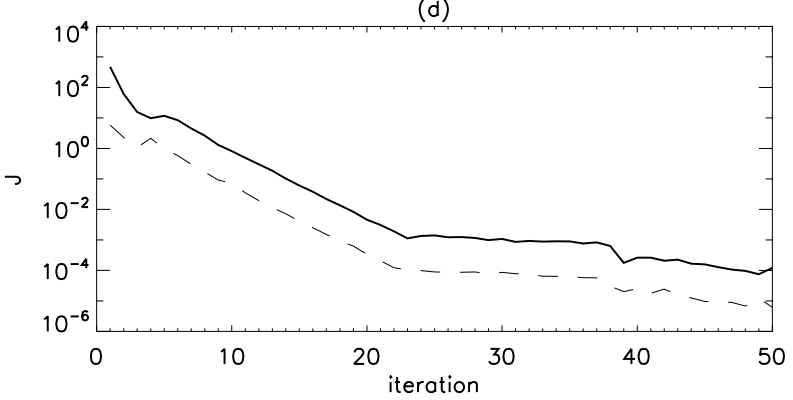

Fig. 5. Results for the initial background from case IV and observations from case V: (a) $\alpha_{\tau}$ versus the number of intervals (note that the number of intervals is equal to the number of sequential minimizations of $J$ ); (b) $R e$ versus the number of intervals; (c) $a$ versus the number of intervals; (d) the initial value of the cost function (drawn), its value after minimization over the initial conditions (dotted) and after minimization over $R e$ (dashed).

solving accurately and efficiently this multi-parameter estimation problem.

In the next test problem, we will use the time-dependent streamfunction field of case VI as observations, while still keeping case IV as background. For case VI, the value of $R e$ is even larger than that of case $\mathrm{V}$ and the jet oscillates around a "jet-down" mean. Note that one parameter, $a$, initially has the correct value but that it is free to vary during the parameter estimation procedure. (a)

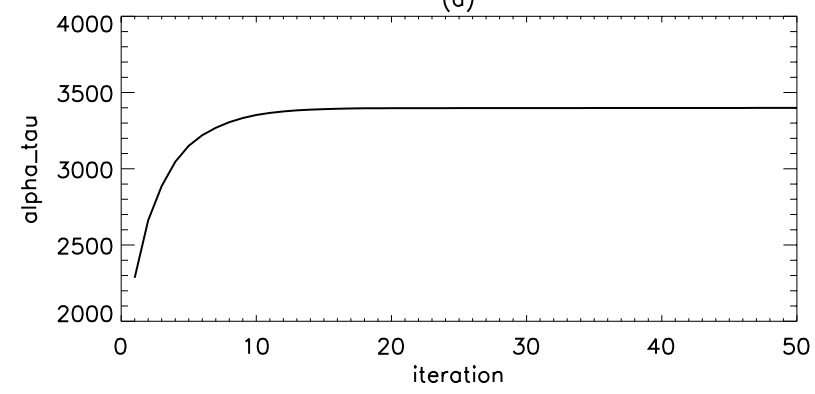

(b)

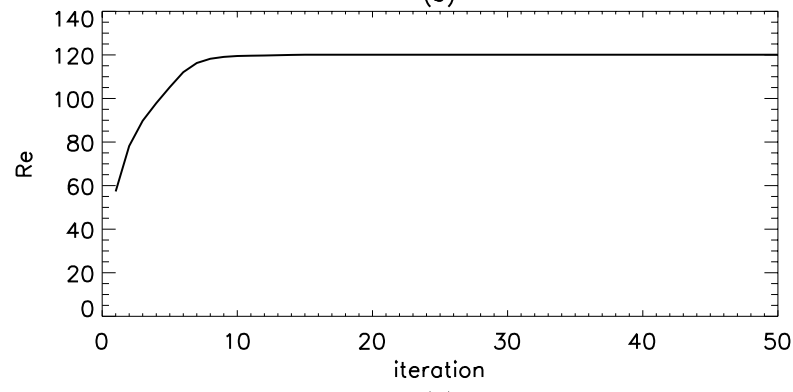

(c)

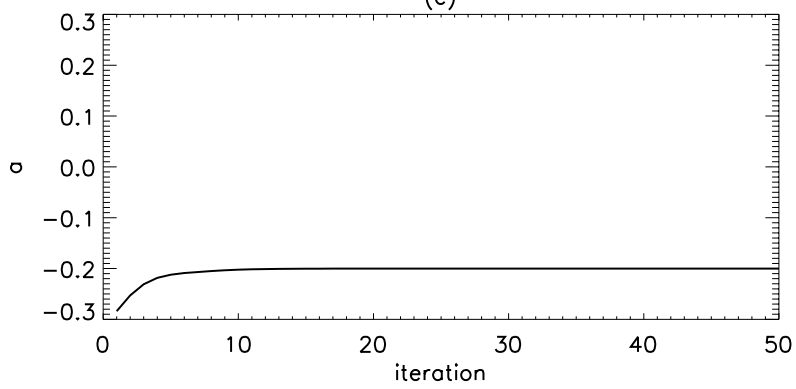

(d)

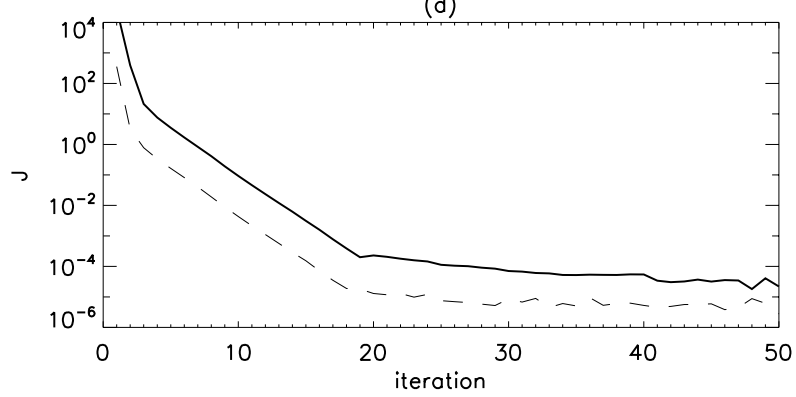

Fig. 6. Results for initial background from regime IV and observations from regime VI: (a): $\alpha_{\tau}$ versus the number of intervals (note that the number of intervals is equal with the number of sequential minimizations of $J$ ); (b) $R e$ versus the number of intervals; (c) $a$ versus the number of intervals; (d) the initial value of the cost function (drawn), its value after minimization over initial conditions (dotted) and after minimization over $R e$ (dashed).

After 8 intervals the estimates for $\alpha_{\tau}$ and $R e$ are already close to the target values of case VI (Fig. 6a, b). The initially correct parameter, $a$, is changed at first, but recovers to its initial value. This indicates that, although values of initially correct parameters may change in the beginning, the final estimates of those parameters are recovered. Compared to the results of the previous test problem, the figures of the behavior of the cost function look qualitatively the same (compare 
Fig. 6d and Fig. 5d). The same rapid decrease is seen in the first 20 intervals, as is the slow convergence thereafter due to the stop criteria. The results of this test problem also shows that I4D-Var gives an analysis close to the observations and an accurate estimation of the parameters in the model.

These two test problems were among several investigated. The other problems investigated involved other combinations of several initial steady state solution and their associated parameters and steady state, (quasi-)periodic and irregular observations. For some of these problems one or two parameters did have the correct value initially. The result were as good as the two multi-parameter estimation problems discussed in this section. Accurate estimations of the parameters were found and the analysis was always close to the observations.

\section{Conclusions}

The main point of this paper was to show that one can perform 4D-Var data assimilation without using an explicit adjoint model, when an implicit forward model formulation is available. In that case, the tangent-linear model, needed for the evaluation of the gradient of the cost function, can be derived and its transpose can be explicitly calculated without much extra cost.

Implicit forward models have an advantage that usually larger time steps can be taken than with explicit forward models. The choice of the time step in implicit models is not limited by numerical stability, like in explicit models, but by numerical accuracy. The discrete derivation of the implicit models is in most cases more complicated than those of explicit models, since the Jacobian matrix has to be obtained and large linear systems of equations involving this matrix have to be solved. Over the last decade a hierarchy of implicit ocean and climate models has been developed, aided by the development of efficient solvers for linear systems of equations (Dijkstra, 2000).

Here, a simple one-layer quasi-geostrophic model of the double-gyre wind-driven circulation was used as background model and for generating observations. For this model, the different flow regimes are known for different values of the control parameters. The I4D-Var method performs well in a variety of test problems for this model, involving both single and multi parameter estimation. Even when the initial background model and the observations are chosen in different dynamical regimes, I4D-Var is able to find an accurate estimate of the uncertain parameters in the model as well as a perfect fit to the observations.

While this is the first step in the development of implicit 4D-Var methods, there are several issues which need further study to evaluate the potential use of these methods in more realistic models and real world applications. These are (i) the effect of noisy observations and more complex behavior of trajectories, and (ii) the effect of an increase in dimension of the state space. While we cannot address these issues here in depth, we discuss each of them briefly below.
A few additional cases were studied to test the performance of I4D-Var under "noisy" observations. We added Gaussian noise, with zero mean and a prescribed standard deviation $\sigma$, to the model-derived observations and considered a single parameter setup with $R e$ as the uncertain parameter. Other parameters had standard values as in Table 1. For the initial background, the symmetric steady state of case I $(R e=20)$ was taken and the observations consisted of the asymmetric steady state from case II $(R e=50)$. For several values of $\sigma$, in the range $0.001-0.2$, a twenty-member ensemble of estimations for $R e$ was calculated. The estimates of $R e$ in the ensemble members decreased when $\sigma$ was increased, but they stabilized between $R e=40$ and $R e=45$. However, the spread around the ensemble mean was significant and the best estimate of $R e$ (for each values of $\sigma$ ) was often around $R e=49$. For large standard deviations, the observed values of $\psi$ close to zero (right side of the basin and around the jet) can change several orders of magnitude and/or sign. This leads to an ill-posed minimization problem or sudden increases in $J$. Although I4D-Var was not able to estimate the value for $R e$ exactly, the method is able to provide values close to the correct value.

Variational methods seem to have a disadvantage when compared to the EnKF method, since they rely on accurate adjoints and gradients. In our methodology, we circumvent constructing the adjoint model, by utilizing the extra information available in the implicit model. In this methodology, we linearize the model at every point of the assimilation interval, which gives a gradient that is more accurate than when an adjoint method was used. This makes the estimation technique more suitable for parameter estimation in nonlinear models. In Lea et al. (2000), some fundamental methodological issues concerning sensitivity analysis of chaotic systems are addressed. They show that, for the Lorenz system, variational methods are a limited tool for sensitivity analysis and parameter estimation, due to the behavior of the adjoint and gradient for various time scales. Note that Lea et al. (2000), however, also found a range of time-scales for which the adjoint was reasonably accurate. This suggests that if the integration segment is chosen carefully, variational methods, such as I4D-Var, may still produce good results.

The potential for real world applications of I4D-Var heavily depends on the quality and performance of numerical solvers of giant dimensional linear systems. With respect to the computational work, which has to be performed in the implicit time stepping, the extra costs of the I4D-Var method are (i) the storage of several Jacobian matrices and (ii) the solution of the additional linear systems. As in most implicit ocean models (Weijer et al., 2003), efficient storage schemes are used for the Jacobian, the extra storage is not expected to become a severe problem for more complex models. In addition, the preconditioners which have been developed for the implicit time stepping procedure can also be used to solve the additional linear systems in I4D-Var. Currently, systems of $\mathcal{O}\left(10^{6}\right)$ equations can be solved with techniques such as MRILU combined with GMRES (Botta and Wubs, 1999) which makes it possible to apply I4D-Var to ocean or atmo- 
sphere models with reasonable resolution. The main advantage of I4D-Var over traditional 4D-Var methods is that at each time step, changes in the state due to a parameter variation are taken into account because of the availability and use of the local Jacobian matrix.

In summary, the results look promising and motivating enough to apply I4D-Var to problems where the dimension of the state space is much larger and where "real" (noisy) observations are used.

Acknowledgements. The authors thank the organizers of the session "Nonlinear Dynamics of Earth, Oceans and Space" at the first AOGS meeting in Singapore (July 2004), F. Wubs and A. de Niet (both University of Groningen, the Netherlands) for the ongoing collaboration, and an anonymous reviewer for very thoughtful comments on the first version of this paper. This work was supported by the Dutch Technology Foundation (STW) within the project GWI.5798.

Edited by: P. C. Chu

Reviewed by: one referee

\section{References}

Anderson, J.: An ensemble adjustment Kalman filter for data assimilation, Monthly Weather Review, 129, 2884-2902, 2001.

Annan, J. and Hargreaves, J.: Efficient parameter estimation for a highly chaotic system, Tellus, 56A, 520-526, 2004.

Annan, J., Hargreaves, J., Edwards, N., and Marsh, R.: Parameter estimation in an intermediate complexity earth system model using an ensemble Kalman filter, Ocean Modelling, 8, 135-154, 2005.

Botta, E. F. F. and Wubs, F. W.: MRILU: An effective algebraic multi-level ILU-preconditioner for sparse matrices, SIAM J. Matrix Anal. Appl., 20, 1007-1026, 1999.

Courtier, P., Thépaut, F.-N., and Hollingsworth, A.: A strategy for operational implementation of 4D-Var, using an incremental approach, Quart. J. Roy. Meteor. Soc., 120, 1367-1388, 1994.

Derber, J.: A variational continuous assimilation method, Monthly Weather Review, 117, 2437-2446, 1989.

Dijkstra, H. A.: Nonlinear Physical Oceanography: A Dynamical Systems Approach to the Large Scale Ocean Circulation and El Niño., Kluwer Academic Publishers, Dordrecht, The Netherlands, 2000.

Dijkstra, H. A. and Katsman, C. A.: Temporal variability of the Wind-Driven Quasi-geostrophic Double Gyre Ocean Circulation: Basic Bifurcation Diagrams, Geophys. Astrophys. Fluid Dyn., 85, 195-232, 1997.

Evensen, G.: Sequential data assimilation with a non-linear quasigeostrophic model using Monte-Carlo methods to forecast error statistics, J. Geophys. Res., 53, 10 143-10 162, 1994.
Evensen, G.: The ensemble Kalman filter: theoretical formulation and practical implementation, Ocean Dynamics, 53, 343-367, 2003.

Hargreaves, J., Annan, J., Edwards, N., and Marsh, R.: An efficient climate forecasting method using an intermediate complexity Earth System Model and the ensemble Kalman filter, Climate Dynamics, 23, 745-760, 2004.

Kalman, R.: A new approach to linear filtering and prediction problems, J. Basic Eng., 82D, 33-45, 1960.

Klinker, E., Rabier, F., Kelly, G., and Mahfouf, J.-F.: The ECMWF operational implementation of four dimensional variational assimilation, Part III: Experimental results and diagnostics with operational configuration, Quart. J. Roy. Meteor. Soc., 126, 11911215, 2000.

Lea, D., Allen, M., and Haine, T.: Sensitivity analysis of the climate of a chaotic system, Tellus, 52A, 523-532, 2000.

Lorenz, E. N.: Deterministic nonperiodic flow., J. Atmos. Sci., 20, 130-141, 1963.

Mahfouf, J.-F. and Rabier, F.: The ECMWF operational implementation of four dimensional variational assimilation. Part II: Experimental results with improved physics, Quart. J. Roy. Meteor. Soc., 126, 1171-1190, 2000.

Nash, S. and Sofer, A.: Linear and nonlinear programming, McGraw-Hill series on industrial and management sciences, McGraw-Hill, 1996.

Navon, I.: Practical and theoretic aspects of adjoint parameter estimation and identifiability in meteorology and oceanography, Dyn. Atmos. Oceans, 27, 55-79, 1998.

Pedlosky, J.: Geophysical Fluid Dynamics. 2nd Edn, SpringerVerlag, New York, 1987.

Rabier, F., Järvinen, H., Klinker, E., Mahfouf, J.-F., and Simmons, A.: The ECMWF operational implementation of four dimensional variational assimilation. Part I: Experimental results with simplified physics, Quart. J. Roy. Meteor. Soc., 126, 1143-1170, 2000.

Vialard, J., Weaver, A., Anderson, D., and Delecluse, P.: Three- and four-dimensional variational assimilation with an ocean general circulation model of the tropical Pacific. Part II: physical validation, Monthly Weather Review, 131, 1379-1395, 2003.

Weaver, A., Vialard, J., Anderson, D., and Delecluse, P.: Three- and four-dimensional variational assimilation with an ocean general circulation model of the tropical Pacific., Part I: formulation, internal diagnostics and consistency checks, Monthly Weather Review, 131, 1360-1378, 2003.

Weijer, W., Dijkstra, H. A., Oksuzoglu, H., Wubs, F. W., and De Niet, A. C.: A fully-implicit model of the global ocean circulation, J. Comp. Phys., 192, 452-470, 2003.

$\mathrm{Yu}, \mathrm{L}$. and O'Brien, J.: Variatational estimation of the wind stress drag coefficient and the oceanic eddy viscosity profile, J. Phys. Oceanogr., 21, 709-719, 1991.

Zhu, Y. and Navon, I.: Impact of parameter estimation on the performance of the FSU global spectral model using its full-physics adjoint, Monthly Weather Review, 127, 1497-1517, 1999. 